Georgian Mathematical Journal

Volume 13 (2006), Number 2, 229-238

\title{
A GENERALIZATION OF BIHARI'S LEMMA FOR DISCONTINUOUS FUNCTIONS AND ITS APPLICATION TO THE STABILITY PROBLEM OF DIFFERENTIAL EQUATIONS WITH IMPULSE DISTURBANCE
}

\author{
SERGIY BORYSENKO, GIOVANNI MATARAZZO, AND MASSIMO PECORARO
}

\begin{abstract}
This paper presents a generalization of nonlinear integral inequalities of the Gronwall-Bellman-Bihari type for discontinuous functions and its application to the investigation of the practical stability of solutions of systems of integro-differential equations with impulse perturbations at fixed moments of time.
\end{abstract}

2000 Mathematics Subject Classification: 34B15.

Key words and phrases: Differential equations with impulse perturbations, integral inequalities.

\section{INTRODUCTION}

Gronwall-Bellman-Bihari inequalities [1]-[3], [31] and their numerous linear and nonlinear generalizations [15]-[17], [19], [20], [22], [23] for continuous and discontinuous functions [4], [28] play an important role in investigating qualitative characteristics of solutions of differential equations such as existence, uniqueness, boundedness, stability with various kinds of perturbations [5]-[13], [21], [22], [24], [25]-[31].

In this paper we establish a new nonlinear integral inequality ("integro-sum" inequality) for discontinuous functions. This term was for the first time used in [28] and subsequently in [9], [10], [30].

In Section 2 we consider the mathematical model of some processes described by a system of integro-differential equations with impulse perturbations at fixed moments of time.

In Section 3 the result of [2], [3], [25] is generalized to the case of discontinuous functions.

In Section 4 the sufficient conditions of boundedness, stability, practical stability [14] of an undisturbed motion system are established for different kinds of nonlinearities on the right-hand side of a system of equations with impulse perturbations.

\section{Mathematical Model}

Let us consider a system of equations of the form 


$$
\begin{gathered}
\frac{d x}{d t}=f(t, x, K[x(t)]), \quad t \neq t_{i}, \\
\left.\triangle x\right|_{t=t_{i}}=I_{i}(x),
\end{gathered}
$$

where $x \in R^{n}, f \in R^{n}, I_{i} \in R^{n}, i=1,2, \ldots, t \geq t_{0} \geq 0$ are defined in the domain

$$
\begin{gathered}
W=\left\{(t, x): t \in J=\left[t_{0}, T\right], \quad T \leq \infty,\|x\| \leq h=\text { const }>0\right\}, \\
\lim _{i \rightarrow \infty} t_{i}=\infty, \quad t_{i-1}<t_{i}, \quad \forall i=1,2, \ldots, \\
K[x(t)]=\int_{t_{0}}^{t} k(t, \tau, x(\tau)) d \tau,\left.\quad \Delta x\right|_{t=t_{i}}=x\left(t_{i}+0\right)-x\left(t_{i}-0\right)=I_{i}\left(x\left(t_{i}-0\right)\right) .
\end{gathered}
$$

The solution $x(t)$ of system (1) is continuous from the left at the points $\left\{t_{i}\right\}$ and has first kind discontinuity at $\left\{t_{i}\right\}$.

Let us assume that $f(t, 0, K[0])=I_{i}(0)=0, \forall i \in N$ and the functions $f, I_{i}, k$ satisfy the following conditions:

i) $\|f(t, x, y)\| \leq l(t)[\|x\|+\|y\|], l(t) \geq 0, \forall t \geq t_{0}$,

ii) $\|k(t, s, x)\| \leq \xi(s)\|x\|^{m}, \forall s: t_{0} \leq s \leq t, \xi(s) \geq 0, m>0$,

iii) $\left\|I_{i}(x)-I_{i}(y)\right\| \leq \beta_{i}\|x-y\|, \forall x, y \in W, \beta_{i}=$ const $>0$ in domain $W$.

Consider the solution $x(t)=x\left(t, t_{0}, x_{0}\right)$ of the Cauchy problem for system (1) with the initial condition $x\left(t_{0}\right)=x_{0}$. It is obvious that

$$
x\left(t, t_{0}, x_{0}\right)=x_{0}+\int_{t_{0}}^{t} f(\tau, x(\tau), K[x(\tau)]) d \tau+\sum_{t_{0}<t_{i}<t} I_{i}\left(x\left(t_{i}-0\right)\right) .
$$

We call (2) the "integro-sum" representation of the solution $x\left(t, t_{0}, x_{0}\right)$.

By virtue of i)-iii), the "integro-sum' inequality for $\|x(t)\|\left(x(t)=x\left(t, t_{0}, x_{0}\right)\right)$ can be written in the form

$$
\begin{aligned}
\|x(t)\| \leq\left\|x_{0}\right\| & +\int_{t_{0}}^{t} l(\tau)[\|x(\tau)\|+\|K[x(\tau)]\|] d \tau+\sum_{t_{0}<t_{i}<t}\left\|I_{i}\left(x\left(t_{i}-0\right)\right)\right\| \\
\leq\left\|x_{0}\right\| & +\int_{t_{0}}^{t} l(\tau)\|x(\tau)\| d \tau+\int_{t_{0}}^{t}\left[l(s)\left(\int_{t_{0}}^{s} \xi(\sigma)\|x(\sigma)\|^{m} d \sigma\right)\right] d s \\
& +\sum_{t_{0}<t_{i}<t} \beta_{i}\left\|x\left(t_{i}-0\right)\right\| .
\end{aligned}
$$

We use estimates for $\|x(t)\|$ to investigate different qualitative characteristics of solutions of system (1). 


\section{The Bellman-Bihari-Rakhmatullina Generalization Method FOR "INTEGRO-SUM" INEQUALITIES}

Using the results of the monograph [30], let us consider the "integro-sum" inequality

$$
u(t) \leq \varphi(t)+\int_{t_{0}}^{t} \Phi(t, s, u(s)) d s+\sum_{t_{0}<t_{i}<t} \Psi\left(t, t_{k}\right) \mu_{k}\left(u\left(t_{k}-0\right)\right),
$$

where $u(t), \varphi(t), \Psi\left(t, t_{k}\right)$ are continuous nonnegative functions for $t \geq t_{0}, u(t)$ having first kind discontinuities at the points $t_{k}$ :

$$
t_{0}<t_{1}<\cdots, \quad \lim _{i \rightarrow \infty} t_{i}=\infty ;
$$

$\Phi(t, s, u)$ is nonnegative at $t \geq s \geq t_{0}$, defined in domain $t \geq s \geq t_{0},|u| \leq$ $k=$ const $<\infty$ and, for fixed $t$ and $s$, it is nondecreasing with respect to $u$; the functions $\mu_{k}(u)$ are continuous nonnegative and nondecreasing with respect to $u$.

Then, for arbitrary $t \in\left[t_{0}, \infty\left[u(t)\right.\right.$ satisfy the inequality $u(t) \leq \sigma_{\varphi}(t)[30$, Theorem 3.1, p. 174], where $\sigma_{\varphi}(t)$ is some solution of the equation

$$
\sigma(t)=\varphi(t)+\int_{t_{0}}^{t} \Phi(t, s, \sigma(s)) d s+\sum_{t_{0}<t_{k}<t} \Psi\left(t, t_{k}\right) \mu_{k}\left(\sigma\left(t_{k}-0\right)\right),
$$

continuous on each intervals $\left[t_{k}, t_{k+1}\left[, k=0,1, \ldots\right.\right.$. Here $\sigma\left(t_{i}-0\right)=\lim _{t \rightarrow t_{i-0}} \sigma(t)$.

The following statement is true.

Lemma. Let the nonnegative function $\varphi(t)$ with first kind discontinuities at the points $t_{i}: t_{1}<t_{2}<\cdots, \lim _{i \rightarrow \infty}=\infty$ satisfy the "integro-sum" inequality

$$
\begin{aligned}
\varphi(t) \leq C & +\int_{t_{0}}^{t} q(s) \varphi(s) d s+\int_{t_{0}}^{t} q(s)\left(\int_{t_{0}}^{s} g(\sigma) \varphi^{m}(\sigma) d \sigma\right) d s \\
& +\sum_{t_{0}<t_{i}<t} \beta_{i} \varphi\left(t_{i}-0\right), m>0,
\end{aligned}
$$

where $C \geq 0, q(t) \geq 0, g(t) \geq 0, \beta_{i}=$ const $\geq 0$.

Then the following estimates are valid:

$$
\text { I) } \begin{aligned}
\varphi(t) & \leq \exp \left[\int_{t_{0}}^{t} q(\tau) d \tau\right]\left[\left\{C \prod_{t_{0}<t_{i}<t}\left(1+\beta_{i}\right)\right\}^{1-m}\right. \\
& \left.+(1-m) \int_{t_{0}}^{t} g(s)\left[\exp (m-1) \int_{t_{0}}^{s} q(\sigma) d \sigma\right] d s\right]^{\frac{1}{1-m}}, \quad 0<m<1
\end{aligned}
$$




$$
\begin{aligned}
& \text { II) } \varphi(t) \leq C \prod_{t_{0}<t_{i}<t}\left(1+\beta_{i}\right) \exp \left[\int_{t_{0}}^{t}(q(\tau)+g(\tau)) d \tau\right], \quad m=1 \\
& \text { III) } \varphi(t) \leq C \prod_{t_{0}<t_{i}<t}^{t}\left(1+\beta_{i}\right) \exp \left[\int_{t_{0}}^{t} q(\tau) d \tau\right]\left[1-(m-1) \prod_{t_{0}<t_{i}<t}\left(1+\beta_{i}\right)^{m-1}\right. \\
&\left.\times C^{m-1} \int_{t_{0}}^{t} g(s)\left(\exp (m-1) \int_{t_{0}}^{s} q(\sigma) d \sigma\right) d s\right]^{-\frac{1}{m-1}}, \quad m>1 \\
& \forall t \geq t_{0}: \int_{t_{0}}^{t} g(s)\left(\exp \left[(m-1) \int_{t_{0}}^{s} q(\sigma) d \sigma\right]\right) d s \\
&<\left[(m-1) \prod_{t_{0}<t_{i}<t}\left(1+\beta_{i}\right)^{m-1} C^{m-1}\right]^{-1}
\end{aligned}
$$

Proof. Suppose that $t \in\left[t_{0}, t_{1}[\right.$. Then

$$
\varphi(t) \leq C+\int_{t_{0}}^{t} q(s) \varphi(s) d s+\int_{t_{0}}^{t} q(s)\left(\int_{t_{0}}^{s} g(\sigma) \varphi^{m}(\sigma) d \sigma\right) d s .
$$

Denote

$$
V(t)=C+\int_{t_{0}}^{t} q(s) \varphi(s) d s+\int_{t_{0}}^{t} q(s)\left(\int_{t_{0}}^{s} g(\sigma) \varphi^{m}(\sigma) d \sigma\right) d s .
$$

It is obvious that $\varphi\left(t_{0}\right)=V\left(t_{0}\right)=C, \varphi(t) \leq V(t), \forall t \geq t_{0}$. Then

$$
\frac{d V}{d t}=q(t) \varphi(t)+q(t) \int_{t_{0}}^{t} g(\sigma) \varphi^{m}(\sigma) d \sigma \leq q(t)\left[V(t)+\int_{t_{0}}^{t} g(\sigma) V^{m}(\sigma) d \sigma\right] .
$$

Let $W(t)=V(t)+\int_{t_{0}}^{t} g(\sigma) V^{m}(\sigma) d \sigma$. Then $W\left(t_{0}\right)=V\left(t_{0}\right)=C, V(t) \leq W(t)$, $\forall t \geq t_{0}$.

It easy to see that

$$
\frac{d W}{d t} \leq q(t) W(t)+g(t) W^{m}(t)
$$

From the latter differential inequality we have the following estimates for $\varphi(t)$ :

$$
\varphi(t) \leq \exp \left[\int_{t_{0}}^{t} q(\tau) d \tau\right]\left[C^{1-m}+(1-m) \int_{t_{0}}^{t} g(\tau)\right.
$$




$$
\begin{aligned}
\times & \left.\exp \left[(m-1) \int_{t_{0}}^{t} q(\sigma) d \sigma\right] d \tau\right]^{\frac{1}{1-m}} \text { for } 0<m<1, \quad t \geq t_{0}, \\
\varphi(t) \leq & C \exp \left[\int_{t_{0}}^{t}(q(\tau)+g(\tau)) d \tau\right], \text { for } m=1, \quad t \geq t_{0}, \\
\varphi(t) \leq & C \exp \left[\int_{t_{0}}^{t} q(\tau) d \tau\right]\left[1-(m-1) C^{m-1} \int_{t_{0}}^{t} g(\tau)\right. \\
& \left.\times \exp \left[(m-1) \int_{t_{0}}^{t} q(\sigma) d \sigma\right] d \tau\right]^{-\frac{1}{m-1}}, \text { for } m>1 \text { and } \\
\forall t \geq & t_{0}: \int_{t_{0}}^{t} g(\tau) \exp \left[(m-1) \int_{t_{0}}^{t} q(\sigma) d \sigma\right] d \tau<\left[(m-1) C^{m-1}\right]^{-1} .
\end{aligned}
$$

From (11) it follows that inequalities (7)-(9) are fulfilled $\forall t \in\left[t_{0}, t_{1}[\right.$.

Using the scheme described in [4] on the interval $\left[t_{k}, t_{k+1}[, k=1,2, \ldots\right.$, and the estimates for the function $\varphi(t)$ on the interval $\left[t_{k-1}, t_{k}[\right.$, we obtain (by induction) estimates (7)-(9) on the entire interval $J$.

\section{Practical Stability by Chetaev}

Now we investigate the problem of practical stability of a trivial solution $x \equiv 0$ of system (1) with different kinds of nonlinearity $f$ on right-hand side of (1).

A trivial solution of system (1) is called practically stable with respect to $(\lambda, \Lambda, J)$ if there exists exist a continuous and monotonously increasing function $\varphi\left(t_{0},\left\|x_{0}\right\|\right)$ with respect to the second argument, such that for an arbitrary solution $x\left(t, t_{0}, x_{0}\right) \neq 0$ of system (1) the estimate

$$
\left\|x\left(t, t_{0}, x_{0}\right)\right\| \leq \varphi\left(t_{0},\left\|x_{0}\right\|\right), \quad \forall t \in J
$$

is valid, where $\varphi\left(t_{0},\left\|x_{0}\right\|\right)<\Lambda$, if only $\left\|x_{0}\right\|<\lambda, \forall t \geq t_{0}, t \in J$. Here $\lambda<\Lambda$, $J=\left[t_{0}, T\right], T \leq \infty$.

A trivial solution of system (1) is called uniformly practically stable with respect to $t_{0}$ relative to present values $(\lambda, \Lambda, J)$, if for an arbitrary nontrivial solution $x\left(t, t_{0}, x_{0}\right)$ of system (1) the estimate $\left\|x\left(t, t_{0}, x_{0}\right)\right\| \leq \varphi\left(\left\|x_{0}\right\|\right), \forall t \in J$ holds, where $\varphi\left(\left\|x_{0}\right\|\right)<\Lambda$, if and only if $\left\|x_{0}\right\|<\lambda$. Here $\varphi(u)$ is a monotonous increasing function with respect to $u$.

Consider the case where the parameter $0<m<1$.

The following result is valid.

Proposition 1. Let for system (1) the following conditions be fulfilled:

a) inequalities i)-iii) are fulfilled for $m \in] 0,1[$; 
b) $\exists \pi\left(t_{0}\right)=$ const $>0$ :

$$
\prod_{t_{0}<t_{i}<t}\left(1+\beta_{i}\right) \leq \pi\left(t_{0}\right)<\infty, \quad \forall t \in J
$$

c) $\exists I_{i}\left(t_{0}\right)=$ const $>0 \quad(i=1,2)$ :

$$
\begin{gathered}
\int_{t_{0}}^{t} l(s) d s \leq I_{1}\left(t_{0}\right), \quad \forall t \in J, \\
\int_{t_{0}}^{s} \xi(\tau) \exp \left[(m-1) \int_{t_{0}}^{\tau} l(\sigma) d \sigma\right] d \tau \leq I_{2}\left(t_{0}\right)<\infty .
\end{gathered}
$$

Then all solutions of system (1) are bounded. If, in addition to to a)-c), the inequality

$$
\exp \left[(1-m) I_{1}\left(t_{0}\right)\right]\left\{\left[\lambda \pi\left(t_{0}\right)\right]^{1-m}+(1-m) I_{2}\left(t_{0}\right)\right\}<\Lambda^{1-m}
$$

holds, then a trivial solution of system (1) is practically stable with respect to $(\lambda, \Lambda, J)$.

Proof. Using (2) and the "integro-sum" inequality (3), it is obvious that an arbitrary solution $x\left(t, t_{0}, x_{0}\right) \neq 0$ of system (1) satisfies the inequality

$$
\begin{aligned}
\left\|x\left(t, t_{0}, x_{0}\right)\right\| \leq & \exp \left[\int_{t_{0}}^{t} l(\tau) d \tau\right]\left[\left\|x_{0}\right\|^{1-m} \prod_{t_{0}<t_{i}<t}\left(1+\beta_{i}\right)^{1-m}+(1-m)\right. \\
& \left.\times \int_{t_{0}}^{t} \xi(\tau) \exp \left[(m-1) \int_{t_{0}}^{s} l(\sigma) d \sigma\right] d \tau\right]^{\frac{1}{1-m}} .
\end{aligned}
$$

Using (13) and the conditions a) $-\mathrm{c}$ ), it is easy to verify that solutions of system (1) are bounded.

Let

$$
\varphi\left(t_{0},\left\|x_{0}\right\|\right) \stackrel{\text { def }}{=}\left[\left\|x_{0}\right\|^{1-m} \pi^{1-m}\left(t_{0}\right)+(1-m) I_{2}\left(t_{0}\right)\right]^{\frac{1}{1-m}} \exp \left[I_{1}\left(t_{0}\right)\right] .
$$

It is obvious that $\varphi\left(t_{0}, \lambda\right)<\Lambda$ if and only if $\left\|x_{0}\right\|<\lambda$ and (12) holds.

Remark 1. If in Proposition $1 \pi\left(t_{0}\right), I_{i}\left(t_{0}\right)$ are independent of $t_{0}$, then a trivial solution is practically stable uniformly with respect to $t_{0}$.

Consider the case where $m=1$. The next result is valid.

\section{Proposition 2.}

I) Let the condition b) of Proposition 1 be fulfilled and inequalities i)-iii) hold for $m=1$. If $\exists I_{3}\left(t_{0}\right)=$ const $>0: \int_{t_{0}}^{t}(l(\tau)+\xi(\tau)) d \tau \leq I_{3}\left(t_{0}\right)<\infty, \forall t \geq t_{0}$, then all solutions of system (1) are bounded. 
II) Assume that part I) of the proposition is fulfilled and the values of the initial and next perturbations of system (1) satisfy the inequality

$$
\frac{\Lambda}{\lambda}>\pi\left(t_{0}\right) \exp \left[I_{3}\left(t_{0}\right)\right]
$$

Then a trivial solution of system (1) is practically stable (uniformly with respect to $t_{0}$ if $I_{3}, \pi$ are independent of $\left.t_{0}\right)$.

The proof of Proposition 2 follows from the "integro-sum" inequality

$$
\begin{aligned}
\left\|x\left(t, t_{0}, x_{0}\right)\right\| \leq & \left\|x_{0}\right\|+\int_{t_{0}}^{t} l(\tau)\left\|x\left(\tau, t_{0}, x_{0}\right)\right\| d \tau \\
& +\int_{t_{0}}^{t} l(s)\left(\int_{t_{0}}^{s} \xi(\sigma)\left\|x\left(s, t_{0}, x_{0}\right)\right\| d \sigma\right) d s \\
\stackrel{\text { lemma }}{\Longrightarrow}\left\|x\left(t, t_{0}, x_{0}\right)\right\| \leq & \left\|x_{0}\right\| \prod_{t_{0}<t_{i}<t}\left(1+\beta_{i}\right) \exp \left[\int_{t_{0}}^{t}[l(s)+\xi(s)] d s\right] \\
\leq & \left\|x_{0}\right\| \pi\left(t_{0}\right) \exp \left[I_{3}\left(t_{0}\right)\right] \stackrel{\text { def }}{=} \varphi\left(t_{0},\left\|x_{0}\right\|\right) .
\end{aligned}
$$

Remark 2. It can be easily verified that when the conditions of Proposition 2 are satisfied, the estimate

$$
\left\|x\left(t, t_{0}, x_{0}\right)\right\| \leq\left\|x_{0}\right\| \prod_{t_{0}<t_{i}<t}\left(1+\beta_{i}\right) \exp \left[\int_{t_{0}}^{t}[l(s)+\xi(s)] d s\right]
$$

implies that a trivial solution of system (1) is stable by Lyapunov.

Now consider the case where $m>1$. The next result is valid.

Proposition 3. Let system (1) satisfy conditions i)-iii) and the inequalities b), c) of Proposition 1 be fulfilled. Then:

A) all solutions of system (1) are bounded;

B) a trivial solution is

1) practically stable if and only if the initial and next perturbations satisfy the inequalities

1) (p.s.), if only values of initial and next perturbations satisfy inequalities

$$
\begin{gathered}
\lambda\left(t_{0}\right) \pi\left(t_{0}\right)<\left[(m-1) I_{2}\left(t_{0}\right)\right]^{-\frac{1}{m-1}}, \\
\exp \left[I_{1}\left(t_{0}\right)\right] \pi\left(t_{0}\right)\left[1-(m-1) \pi^{m-1}\left(t_{0}\right) \lambda^{m-1}\left(t_{0}\right) I_{2}\left(t_{0}\right)\right]^{-\frac{1}{m-1}}<\Lambda ;
\end{gathered}
$$

2) uniformly practically stable if (16) holds and $I_{i}\left(t_{0}\right)=I_{i}, \pi\left(t_{0}\right)=\pi$, $\forall t_{0} \geq 0$ (independent of $\left.t_{0}\right)$;

C) a trivial solution is stable by Lyapunov if and only if 


$$
\begin{aligned}
\int_{t_{0}}^{t} \xi(s) \exp ((m & \left.-1) \int_{t_{0}}^{s} l(\sigma) d \sigma\right) d s \\
& <\frac{1}{(m-1) \prod_{t_{0}<t_{i}<t}\left(1+\beta_{i}\right)^{m-1}\left\|x_{0}\right\|^{m-1}} \quad \forall t \geq t_{0} \geq 0
\end{aligned}
$$

Statements A)-C) follow from the estimate of the norm of a solution of system (1)

$$
\begin{aligned}
\left\|x\left(t, t_{0} x_{0}\right)\right\| \leq & \left\|x_{0}\right\| \prod_{t_{0}<t_{i}<t}\left(1+\beta_{i}\right)\left[1-(m-1) \prod_{t_{0}<t_{i}<t}\left(1+\beta_{i}\right)^{m-1}\left\|x_{0}\right\|^{m-1}\right. \\
& \left.\times \int_{t_{0}}^{t} \xi(s) \exp \left((m-1) \int_{t_{0}}^{s} l(\sigma) d \sigma\right) d s\right]^{-\frac{1}{m-1}} \exp \left(\int_{t_{0}}^{t} l(\tau) d \tau\right),
\end{aligned}
$$

where $t \geq t_{0}$, and therefore inequality (17) is satisfied.

\section{ACKNOWLEDGEMENT}

The authors would like to thank very much Ms. Helen Somova, the linguist at the Editorial office, Georgian Math. J., for her efforts to improve English in the paper.

\section{REFERENCES}

1. N. V. AzBelev and Z. B. Calyuk, On Čaplygin's problem. (Russian) Ukrain. Mat. Ž. 10(1958), No. 1, 3-12.

2. R. Bellman, Stability theory of differential equations. McGraw-Hill Book Company, Inc., New York-Toronto-London, 1953; Russian transl.: Izdat. InostrannoǐLit., Moscow, 1954.

3. I. Bihari, A generalization of a lemma of Bellman and its application to uniqueness problems of differential equations. Acta Math. Acad. Sci. Hungar. 7(1956), 81-94.

4. S. D. Borisenko, Asymptotic stability of solutions of systems with impulse action. (Russian) Ukrain. Mat. Zh. 35(1983), No. 2, 144-150.

5. S. D. Borisenko, Stability of solutions with respect to a linear approximation of systems with impulse action. (Russian) Differentsial'nye Uravneniya 22(1986), No. 5, 884-886, 918.

6. S. D. Borisenko, Practical stability of solutions of impulsive systems. (Russian) Izv. Vyssh. Uchebn. Zaved. Mat. 1989, No. 1, 14-25; English transl.: Soviet Math. (Iz. VUZ) 33(1989), No. 1, 15-26.

7. S. D. Borisenko, Technical Stability systems with impulse. Proceeding IMACS-IFAC Symposium "Nodelling and Control of Technological Systems", vol. 3, 38-41, Lille, France, May 7-10, 1991.

8. S. D. Borisenko, Construction of Mathemetical Models. Vipol, Kiev, 1995.

9. S. D. Borisenko, On integro-sum functional inequalities. Differential Equations 34 (1998), No. 6, 850. 
10. S. D. Borisenko, On some Bihari inequalities for discontinuous functions. Naukovi Visti NTUU "KPI" 1998, No. 1, 147-151.

11. S. D. Borisenko, V. I. Kosolapov, and A. Yu. ObolenskiI, Stability of processes under continuous and discrete perturbations. (Russian) Naukova Dumka, Kiev, 1988.

12. S. Borysenko, E. Laserra, G. Matarazzo, and M. Pecoraro, About some hyperbolic systems with impulse influence. Int. Conf. "VI Bogolyubov's Readings". Abstract. Kyiv, 2003, p. 266.

13. S. Borysenko, G. Matarazzo, E. Laserra, and M. Pecoraro, Estimates of solutions some hyperbolic equtions with impulse influence. Proc. "X Int. Kravchuk M. Conf.", Kiev, 2004.

14. N. G. Chetaev, Stability of motion. (Russian) 2d ed. Gosudarstv. Izdat. Tehn.-Teor. Lit., Moscow, 1955.

15. S. C. Chu and F. T. Metcalf, On Gronwall's inequality. Proc. Amer. Math. Soc. 18(1967), 439-440.

16. S. G. Deo and M. G. Murdeshwar, A note on Gronwall's inequality. Bull. London Math. Soc. 3(1971), 34-36.

17. U. D. Dhongade and S. D. Deo, Some generalizations of Bellman-Bihari integral inequalities. J. Math. Anal. Appl. 44(1973), 218-226.

18. N. KRYloff and N. Bogoliouboff, La théorie générale de la mesure dans son application à l'étude des systèmes dynamiques de la mécanique non linéaire. Ann. of Math. (2) 38(1937), No. 1, 65-113

19. V. Lakshmikantham and S. Leela, Differential and integral inequalities: Theory and applications. Vol. I: Ordinary differential equations. Mathematics in Science and Engineering, Vol. 55-I. Academic Press, New York-London, 1969.

20. V. Lakshmikantham, A variation of constants formula and Bellman-Gronwall-Reid inequalities. J. Math. Anal. Appl. 41(1973), 199-204.

21. A. A. Martynyuk and S. D. Borisenko, Technical stability on an unbounded interval. (Russian) Mat. Fiz. No. 28 (1980), 22-28, 122.

22. A. A. Martynyuk, V. Lakshmikantam, and S. Leela, Stability of motion: the method of integral inequalities. (Russian) Naukova Dumka, Kiev, 1989.

23. B. G. Pachpatte, A note on Gronwall-Bellman inequality. J. Math. Anal. Appl. 44(1973), 758-762.

24. V. Raghavendra and M. Rama Mohana Rao, On the stability of differential systems with respect to impulsive perturbations. J. Math. Anal. Appl. 48(1974), 515-526.

25. L. F. Rakhmatullina, An application of the condition of solvability of Caplygin's problem to the question of boundedness and stability of solutions of differential equations. Izv. Vyš̌. Učebn. Zaved. Matematika 1959, No. 2 (9), 198-201.

26. M. Rama Mohana Rao, A note on an integral inequality. J. Indian Math. Soc. (N.S.) 27(1963), 67-69.

27. A. N. V. RAO and C. P. Tsokos, On the existence of a random solution to a nonlinear perturbed stochastic integral equation. Ann. Inst. Statist. Math. 28(1976), No. 1, 99-109.

28. A. M. SamoÍlenko and S. D. Borisenko, Integro-summed inequalities and the stability of processes with discrete perturbation. (Russian) Differential equations and applications, I, II (Russian) (Ruse, 1985), 377-380, 'Angel Kanchev' Tech. Univ., Ruse, 1987.

29. A. M. Samollenko and S. D. Borysenko, On functional inequalities of Bihari type for fiscontinuous functions. Uspekhi Mat. Nauk 53(1998), No. 4, 147-148. 
30. A. Samoilenko, S. Borysenko, C Cattani, G. Matarazzo, and V. Yasinsky, Differential models. Stability, inequalities \& estimates. Naukova Dumka, Kiev, 2001.

31. A. Samoilenko, S. Borysenko, E. Laserra, and G. Matarazzo, Wendroff type integro-sum inequalities and applications. Math. Notes (Miskolc) 3(2002), No. 2, 123132 .

(Received 7.04.2005)

Authors' addresses:

S. Borysenko

Department of Differential equations

National Technical University of Ukraine "KPI"

Peremogy Prosp. 37, 03056, Kyiv

Ukraine

E-mail: borys@mbox.com.ua

G. Matarazzo, M. Pecoraro

Department of Engineering, Informatics

and Applied Mathematics

University of Salerno,

Ponte Don Melillo, 84084, Fisciano (Salerno)

Italy

E-mail:matarazz@diima.unisa.it 